\title{
Mesure locale dans les écoulements diphasiques: développements récents des sondes optiques
}

\author{
Local measurement in two-phase flow : \\ recent optical probe design developments
}

\author{
François Danel ${ }^{*}$
}

Introduction

L'importance de la connaissance aussi fine que possible de la structure locale des écoulements diphasiques a donné naissance à une instrumentation spécifique permettant en particulier de connaitre en un point de l'écoulement, la nature de la phase présente en fonction du temps.

Les travaux effectués ces dernières années $[1,2]$ ont montré l'intérêt des sondes optiques, leur performance et la variété de leur domaine d'utilisation. Nous nous sommes attachés à étendre leurs possibilités, en particulier vers le domaine des écoulements finement dispersés et vers le domaine des hautes pressions et hautes températures. Nous résumons ici les efforts entrepris ainsi que les résultats obtenus.

\section{Généralités}

Fonction caractéristique de phase

Il s'agit de caractériser la phase en présence à un instant et en un point d'un écoulement diphasique. A cet effet, on définit la fonction de phase comme une fonction binaire prenant en un point la valeur zéro lorsque la phase présente en ce point est la phase liquide, et la valeur unité lorsqu'il s'agit de la phase gazeuse $\Psi_{K}=1$ si en présence de la phase $K$

$\Psi_{K}=0$ si ce n'est pas le cas

\section{$K$ gaz ou liquide}

On montre [2] que la connaissance de cette fonction dans l'espace et le temps caractérise les configurations d'écoulement.

(*) Assistance Industrielle Dauphinoise, Zirst, 38240 Meylan.
Qualité des mesures

Une sonde idéale permet de déterminer la fonction de phase de manière ponctuelle et instantanée ; sa présence ne modifie pas l'écoulement dans la zone de mesure. Une sonde réelle s'écarte de ces conditions idéales et tout l'effort de l'expérimentation consiste à corriger ces défauts, soit directement par la conception même de la sonde, soit à posteriori, par le traitement du signal.

Certains défauts physiques sont inhérents à chaque type de sonde, et nous y reviendrons dans la suite. La taille non négligeable du volume de mesure est, à des degrés divers, commune à tous les types de sonde. De plus, par sa présence au sein de l'écoulement, la sonde provoque une perturbation que l'on doit minimiser.

Conformité entre la grandeur mesurée et celle introduite dans la modélisation des écoulements

Par abus de langage, on parle souvent de sonde de taux de vide alors, qu'en fait, la sonde mesure la fonction de phase (le taux de vide se définit comme l'intégration dans un domaine ou dans un temps de la fonction de phase).

De même, on utilise dans de nombreux modèles mathématiques la vitesse d'interface $V i$ comme paramètre fondamental, cette vitesse étant définie suivant la normale à la surface [13], tandis qu'à l'aide de deux sondes proches, on mesure un temps de transit dont l'inverse est proportionnel à $V i \operatorname{Cos} \theta, \theta$ étant l'angle entre la normale et l'interface et la droite joignant l'extrémité des deux sondes servant à la mesure. Certains auteurs ont proposé des modèles statistiques permettant la détermination de $V i$ à partir du temps de transit. 
Principaux procédés utilisés pour mesurer la fonction de phase

On utilise la modification d'une propriété physique pour caractériser le changement d'une phase à l'autre. Les procédés ayant donné lieu à des applications utilisent les propriétés électrique, thermique ou optique des fluides.

\section{Sondes électriques}

La variation de la résistance entre deux électrodes placées au sein du fluide détermine la phase présente dans la zone entourant ces électrodes; la géométrie des électrodes conduit, pour éviter les effets de polarisation, à utiliser des courants très faibles, ce qui rend très difficile la protection contre les parasites électriques. Certains auteurs $[11,15]$, ont mis au point des montages électroniques permettant de s'affranchir de ces problèmes, tout en gardant une bonne réponse en fréquence.

Cependant, les effets de film liquide retenu par la partie sensible de la sonde lors d'un passage vers la phase gazeuse retarde la détection du changement de phase. Ce défaut très important peut être en partie compensé par l'electronique de traitement mais cette compensation dépend du type d'écoulement et doit être effectuée par un étalonnage fastidieux, (Fig. 1). De plus, par son principe, cette sonde ne peut etre utilisée dans des fluides isolants.

On a également essayé d'utiliser la variation de la constante dielectrique pour détecter le changement de

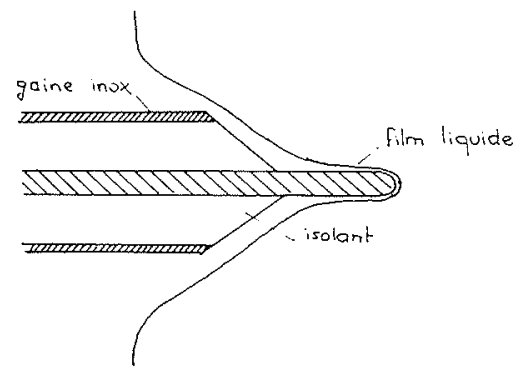

Figure 1 phase, mais il est très difficile de limiter le volume de mesure à une dimension raisonnable sans utiliser des fréquences très élevées.

\section{Sondes thermiques}

\section{a) Microthermocouples [4,19]}

Une sonde comportant un microthermocouple $(20 \mu \mathrm{m})$ permet la mesure locale de la température. Si la jonction de ce thermocouple n'est pas isolée du fluide, on peut détecter la phase en présence par une mesure de conductivité

Cette méthode qui nécessite une électronique soignée pour séparer le signal de température du signal de phase permet de séparer l'histogramme en phase vapeur de celui en phase liquide [4], (Fig. 2).

\section{b) Film chaud}

Une couche métallique mince déposée à la surface d'un support isolant et elle-même isolée du fluide ambiant, est chauffée par effet Joule. Suivant la vitesse et la nature de la phase en présence, le flux thermrue entraîné par le fluide varie et modifie la température et la résistance de la sonde. On accède ainsi en principe à la fonction de phase ainsi qu'à la vitesse de chacune des phases [8]. Delhaye a montré la complexité de l'interprétation du signal lors du passage des interfaces (Fig. 3).

\section{Sondes optiques}

On utilise la variation de l'indice de réfraction du milieu ce qui caractérise le changement de phase. Ce principe utilisé depuis longtemps pour la détection des niveaux a donné lieu à plusieurs réalisations $[2,9$, 10]. Pour pallier aux défauts des sondes précédentes en ce qui concerne la détection de présence des phases et détecter de façon plus précise le passage des interfaces, Danel/Delhaye ont développé un nouveau type de sonde qui a permis d'allier des performances intéressantes et une miniaturisation de sa partie sensible [3].

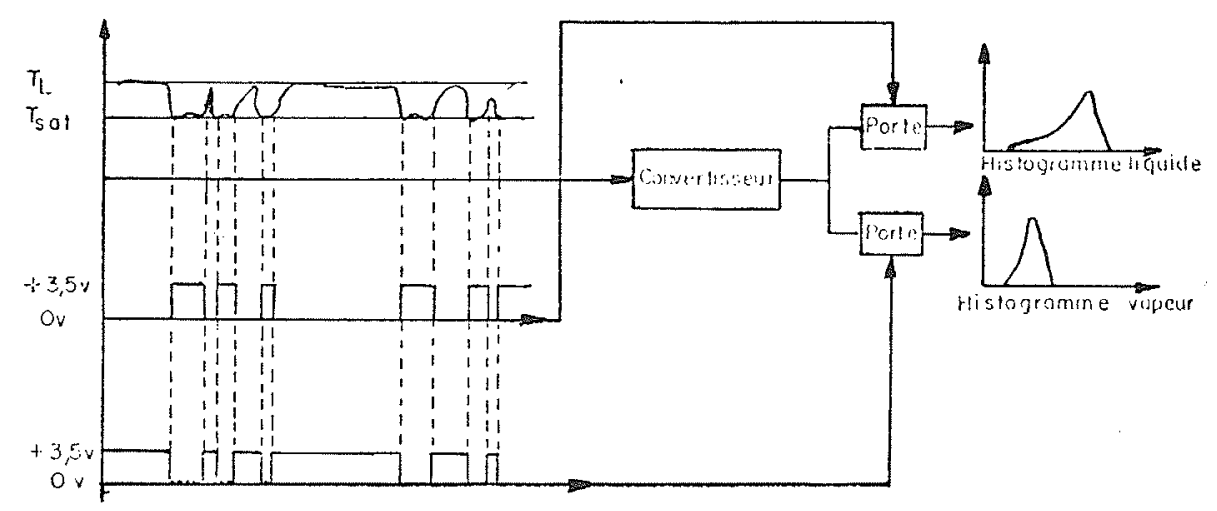

Figure 2 

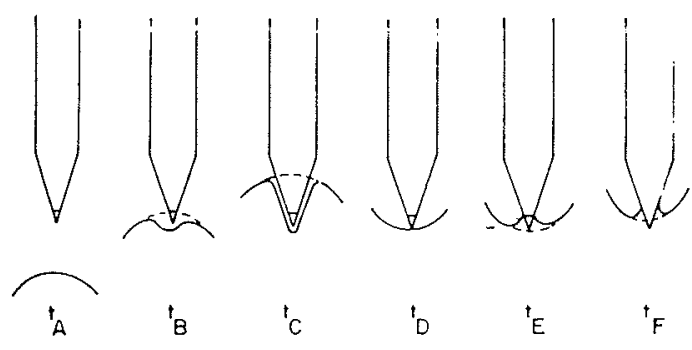

${ }^{t} B$

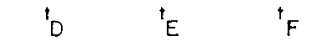

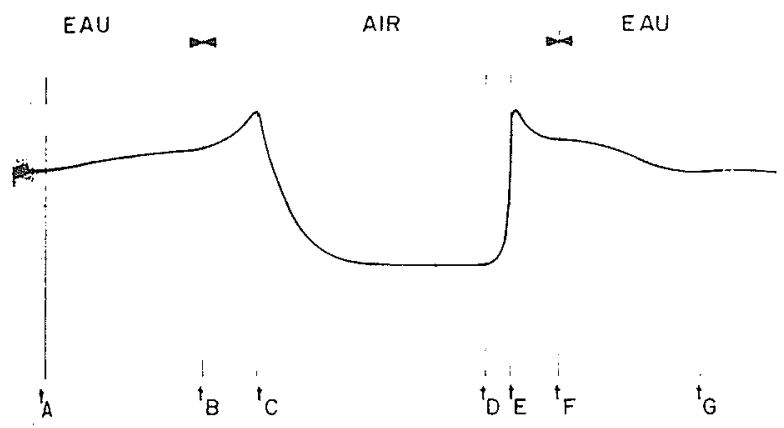

Figure 3
Dès 1969, il est apparu que l'utilisation des fibres optiques pourrait donner naissance à des instruments ayant des performances très supérieures à celles apportées par les autres procédés, et plus particulièrement, on pourrait envisager des mesures dans des phases non conductrices comme le fréon qui prendrait alors une grande importance pour la simulation à basse température de l'eau pressurisée. De plus, on pourrait espérer supprimer les problèmes de parasites électriques particulièrement critiques lorsque l'on doit manipuler des signaux faibles dans une ambiance de courant fort telle que celle de canaux chauffés électriquement. Après divers essais, ces recherches ont donné lieu à plusieurs réalisations dont la première est commercialisée depuis plusieurs années et dont les autres sont en cours de qualification en laboratoire.

Le principe de base des méthodes utilisées est toujours le même (Fig. 4). On mesure la lumière réfléchie entre deux milieux, la sonde et la phase en contact. Pour un indice donné de l'extrémité de la sonde de l'ordre

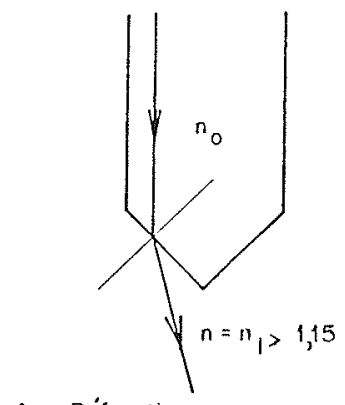

A - Réfracion

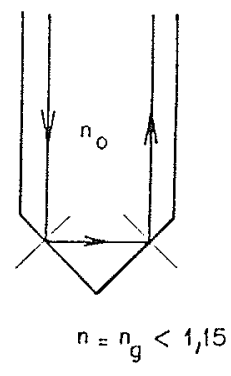

B_Réflexion totale
Figure 4 de 1,5 on passe, pour un angle d'incidence de l'ordre de $45^{\circ}$, progressivement d'une transmission presque totale à une réflexion totale lorsque l'indice s'abaisse de celui du liquide à celui du gaz.

Des considérations concernant la géométrie d'extrémité, les couplages parasites ont fait choisir diverses solutions répondant aux problèmes rencontrés.

\section{Sonde en U Danel/Delhaye (Fig. 5)}

Elle utilise une fibre optique à gradient transverse d'indice pour assurer une bonne transmission de la lumière entre la source, la partie sensible et le photorécepteur. Une fibre monobrin est utilisée pour l'éclairage, l'autre pour la réception. On obtient ainsi un très bon rapport signal sur bruit. La partie sensible est constituée par la fibre repliée en U sur elle-même, le fort rayon de courbure permet à la lumière de traverser les couches d'indice de la fibre et d'atteindre l'interface verre/liquide et d'effectuer une mesure de présence de la phase en contact.

Cette conception conduit à une sonde de faible dimension $(100 \mu)$ ayant un temps de réponse limité uniquement par le temps de montée du photorécepteur $(50 \mu \mathrm{s})(1)$.

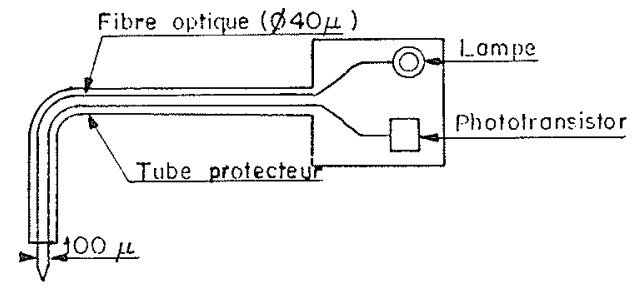

Figure 5

La sonde de $U$ de Danel/Delhaye a permis de nombreux essais et reste suffisante dans le plupart des cas. Cependant, la demande pour des études dans des écoulements finement dispersés conduit à diminuer encore la taille de l'extrémité de la sonde, de même que les problèmes posés par la tenue aux températures et pressions élevées en présence d'eau déminéralisée ont conduit à développer de nouveaux procédés.

\section{Sonde monofibre (3) (Fig. 6)}

Dans cette réalisation, on utilise la même fibre pour l'éclairage et pour la réception du signal, l'extrémité de la fibre étant taillée en pointe. On obtient ainsi une

(1) Une variante a été proposée récemment par Jones [19], dans laquelle la jonction est polie en forme de dièdre, et protégée jusqu'à sa partie sensible par la gaine métallique. Il semble qu'elle donne des résultats dépendants de la vitesse de traversée des interfaces, l'électronique introduisant aussi des dépassements non totalement expliqués, venant perturber le traitement du signal. Jones a analy sé les défauts possibles des sondes optiques, défauts que l'on ne retrouve pas dans les sondes en U de Danel/ Delhaye, ni đans les sondes qui seront décrites ci-après. 


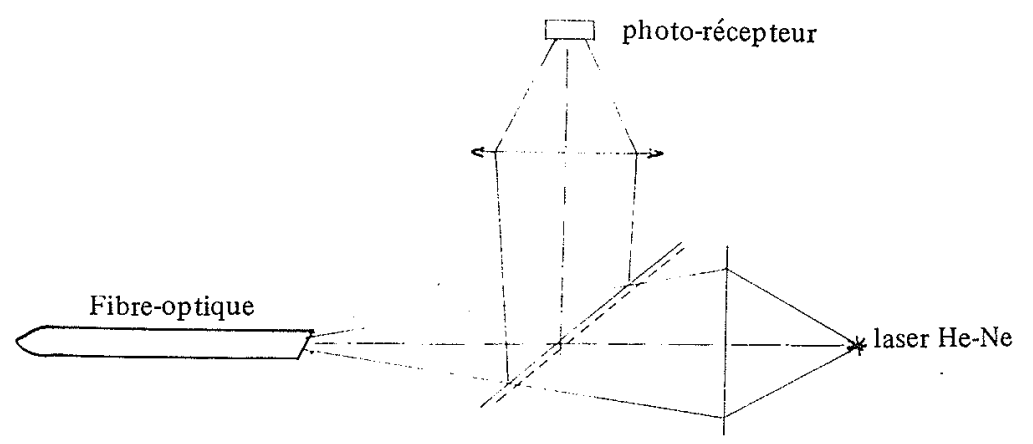

Figure 6 - Schéma d'une sonde monofibre

extrémité d'un diamètre inférieur à $40 \mu$ et profilée, ce qui est très favorable lors de la traversée des interfaces. A l'extrémité opposée à la partie sensible, la fibre est coupée perpendiculairement à son axe, un système de séparatrice permet d'éclairer la fibre et de récupérer le signal. Comme source de lumière, on utilise un petit laser $\mathrm{HeNe} 0,5 \mathrm{~mW}$. La qualité du signal reçu a permis d'utiliser un photorécepteur très rapide, le temps de montée pouvant alors être de l'ordre de la micro-seconde. Le faible bruit et la résolution élevés permettent de distinguer des variations d'indice faible du milieu en contact. On peut alors utiliser cette sonde en liquideliquide, ce qui élargit nettementson champ d'application.

La sonde monofibre ouvre aussi le champ des écoulements à phase liquide finement dispersée où l'on ne disposait pas de sondes utilisables.

\section{Sonde haute température/haute pression [3]}

Les sondes précédentes peuvent fonctionner à des températures et sous des pressions élevées si le fluide n'est pas chimiquement agressif vis-à-vis de l'extrémité de la sonde. Malheureusement, l'eau qui est le fluide le plus utilisé dans les expériences et dans les applications industrielles dissout rapidement les verres (dans les conditions des PWR par exemple), spécialement si cette eau est déminéralisée, ce qui est généralement le cas. Les matériaux qui pourraient constituer l'extrémité de la sonde doivent être transparents, pouvoir être mis en forme et fixés de manière étanche à l'extrémité d'une sonde en métal compatible avec les conditions d'emploi, en général acier inoxydable et titane. On a retenu le diamant et le saphir ; le saphir étant plus facile à travailler on a réalisé des pointes d'extrémités dans ce matériau que l'on a soudé sur des tubes en titane. On obtient des sondes dont la partie sensible fait de l'ordre de 0,4 millimètre à l'extrémité d'un tube de $\emptyset 3$ millimètres.

A l'intérieur du tube, des fibres classiques assurent le couplage optoélectronique entre la partie sensible, la source et le photorécepteur.

On a actuellement testé la réponse optique à froid, la tenue de la liaison saphir-titane à chaud ; un dispositif d'essai global dans les conditions des PWR est en cours de réalisation.
R.T. Lahey a pu montrer l'excellente réponse de ce type de sonde, bien que n'ayant pas encore pu réaliser des expériences dans les conditions extrêmes envisagées [15].

\section{Résultats obtenus avec les sondes optiques}

Le signal obtenu à partir des sondes optiques est très proche du signal théorique cherché comme l'a montré M. Galaup [12]. On a cherché à mieux comprendre comment varie le signal en fonction de la position relative de l'extrémité de la sonde et de l'interface. On s'aperçoit que, contrairement aux sondes conductives, le retard dû à l'entraînement d'un film mince sur l'extrémité de la sonde ne procure pas de distorsion du signal. En effet, la réflexion totale a alors lieu à l'interface liquide gaz, qui est très proche de l'extrémité de la sonde ; le film étant très mince, la géométrie de l'extrémité de la sonde monofibre limite encore l'entrainement du film.

Pour effectuer des traitements des signaux permettant d'accéder au taux de vide, à la vitesse des interfaces... etc., on doit obtenir un signal correspondant le plus exactement à la fonction caractéristique de phase. Un conditionneur de signal compatible avec toutes les sondes optiques et avec certaines sondes conductives a été développé.

Il mémorise en permanence le niveau "liquide" et "gaz" et génère un signal logique de sortie, déclenché par un seuil fixe en pourcentage entre le niveau liquide et le niveau gaz. Un seuil différent peut être réglé pour le passage du liquide vers le gaz et pour le passage du gaz vers le liquide: De plus, un système de validation du signal permet de retrouver la phase en présence, même dans le cas où un des seuils n'a pas été atteint. On respecte ainsi l'alternance obligée des phases.

\section{Conclusion}

Les sondes optiques ont déjà prouvé leur grande facilité d'utilisation et la qualité des mesures obtenues. Les possibilités offertes par les nouveaux types de sonde, monofibre et haute température/haute pression permettront d'étendre le domaine d'utilisation de ces sondes, non seulement dans le domaine liquide/gaz mais aussi dans les écoulements liquide/liquide. 


\section{Références}

[1] GALAUP J.P. et DELHAYE J.M. - Utilisation de sondes optiques miniatures en écoulement diphasique gaz-liquide. La Houille Blanche, $\mathrm{n}^{\circ} 1,1976$.

[2] DANEL F. et DELHAYE J.M. - Sonde optique pour la mesure du taux de présence local en écoulement diphasique. Mesure, régulation, automatisme (août-septembre 1971 p. 99-101).

[3] Les sondes et électroniques de traitement décrites sont fabriquées par A.I.D. (Assistance Industrielle Dauphinoise) et commercialisées hors de France par DISA Electronique.

[4] DELHAYE, SEMERIA, FLAMAND - Mesure du taux de vide local et des températures du liquide et de la vapeur en écoulement diphasique avec changement de phase à l'aide d'un microcouple, CEA.R.4302.1972.

[5] LECROART, PORTE - Electrical probes for study of two-phase flow at high velocity. International symposium on two-phase system, Haifa Israël 1971, paper 3.11.

[6] RESCH F., COANTIC M. -- Etude sur le fil chaud et le film chaud dans l'eau. La Houille Blanche, $\mathrm{n}^{\circ}$ 2, 1969, p. 151-161.

[7] DELHAYE J.M. - Anémométrie en écoulement diphasique, Métrologie des écoulements diphasiques. Quelques procédés. CEA.R. 4457,1973 , p. 38-54.

[8] DELHAYE J.M. - Hot film panemometry in two-phase flow. Two-phase flow instrumentation. ASME, 1969, 58-59.

[9] MILLER, MITCHIE - The development of an universal probe for measurement of local voidage in liquid/gaz two phase flow systems. "Two-phase flow instrumentation".
$A S M E$, Le Tourneau (B.W.) and Bergles (A.E.) ed. 1969 p. 82-88.

[10] HINATA S. - A study on the measurement of the local void fraction by the optical fiber glass probe. Bulletin of J.S. M.E., vol. $15, \mathrm{n}^{\circ} 88, \mathrm{p} .1228-\mathrm{i} 235$.

[11] REVOREUSE M., FLAMAND J.C. - Etude de l'utilisation des sondes résistives dans des écoulements diphasiques à grande vitesse. CENG, rapport TT. n ${ }^{\circ} 111,1972$.

[12] GALAUP J.P. - Contribution à l'étude des méthodes de mesure en écoulement diphasique. Thèse soutenue le 28 avril 1975. Université Scientifique et Médicale de Grenoble et Institut National Polytechnique de Grenoble.

[13] DELHAYE J.M. - Proceeding of the NAID. Advanced Study Institute in two-phase flow and heat transfer. Istambul 1976.

[14] DANEL F. - Two-phase flow measurements. Fluid Dynamic Institute. Short course 78.1.

[15] Dr. LAHEY R.T. - Two-phase flow measurements. Fluid Dynamic Institute. Short course 78-1.

[16] OWEN C. JONES Jr. and DELHAYE J.M. - Transcient and statistical measurement technics for two-phase flow. A critical review. Multiphase flow, Vol. 3 p. 89-116.

[17] DELHAYE J.M. and GALAUP J.P. - Hot film. Panemometry in air water flow. Fourth Biennal Symposium on Turbulence in liquids. Rolla Missouri 1975.

[18] DELHAYE J.M., SEMERIA R., FLAMAND J.C. - Void fraction and vapor liquid temperatures. Local measurements in two-phase flow using a microthermocouple. Joumal of Heat transfert, August 1973.

[19] ABUAF N., JONES O.C. Jr. and ZIMMER G.A. - Optical probe for local void fraction and interface velocity measurement. Review Sci. Instrum. 49 (8) August 1978. 